\title{
Structure of the Earth Natural Geoinfromation System
}

\author{
A.D. Kitov * \\ V.B.Sochava Institute of Geography SB RAS, Irkutsk, Russia
}

\begin{abstract}
The model of geographical space of the Earth is represented as a multilevel discrete grid structure. This provides a scientific basis for the construction of a natural geoinformation system of the globe with invariant organization of data in the form of a universal grid, i.e. a different-level set of elementary cells with discrete topology. A grid structure is a multilevel system of reference points, with respect to which a spatial analysis of the territory is carried out. Reference points have attributes reflecting the territorys hierarchic level and location characteristics. It is found that there is a connection between the topology of the cells and specific structures of the Earth, and between the lineament network and the hydrological network in particular.
\end{abstract}

Key words: geoinformation system, grid structure, geographical network, modelling of geospace, spatial organization of territory, model of geodata presentation, lineament network.

AMS subject classification: 91D20

\section{Introduction}

A gridded model of geographical coordinates tied to a particular cartographic projection of the Earths surface is customarily used in geometric models of the Earth to solve geographical problems and for the purposes of spatial orientation. A typical example is provided by an arbitrary partition of the Earths surface through a graticule of meridians and parallels. There are also other models for representation mapping of space and coordinates, such as the UTM rectangular system or the Gauss-Kruger projection accepted in Russia. In this case, the trapezium between neighbouring meridians and parallels is divided into a network of identical rectangles, and the central meridian of the trapezium is considered as the origin of coordinates. Such a model is not associated with the Earths real structure, and it is impossible to consider it as a natural representation of geographical knowledge. Is it possible to identify the characteristic structural features of the

\footnotetext{
*E-mail: kitov@irigs.irk.ru
} 
globe which determine the natural network of reference points and cells on the Earths surface? The principles of natural hierarchic partition of the Earths surface by introducing the triangulation network and the network of coordinates were discussed earlier by A. Cherkashin [1]. Such a reference network serves as a basis for the construction of the geoinformation system (GIS) model to solve geographical tasks using the natural spatial characteristics of the globe.

The further development of GIS technology-based geoinformation mapping must involve a systematization of geographical information accumulated in a GIS and the creation of models for presentation of spatial data on a general natural basis where the structure of the GIS database reflects the laws of organization of geospace a distinctive, objectively existing geoinformation system of the Earth. In this case, computer processing of data, such as remotely sensed data, must take into consideration not only the local features of the location of the object but also its arrangement in a global network of information coordination related to a classification of geographical complexes (the knowledge base) forming the basis for formulating inferences and identifying polytypic models for a problem or a situation in the interests of effective territorial management.

Such a problem statement focuses on a continuation of research on the generation territorial GIS within the framework of the general research area Integrated GIS of Territorial Management [2] and is aimed at the creation of evolving geoinformation systems that are independent of the location, the form of structural mapping of territorial objects, spatio-temporal scales, the content of current tasks, and of the methods of addressing them. The problem of combining and unifying raster and vector GIS data and generating of an invariant spatial structure of GIS data presentation is discussed.

\section{Models and methods}

At present, grid rasters of digital space geoimages, vector topographic and landscape maps, forest management plans with an irregular network of mapping units, a compartment network of forest management, administrative zoning of the territory etc. are used in GIS as a structure-invariant basis of thematic mapping and modelling. To solve scientific and applied problems demands further universalization and unification of presentation of these invariant bases interconnected with the GIS database in the form of combined (vector-raster) grid structures of multilayer polymatrices [3].

It seems appropriate to tackle this problem by generating an invariant structure of GIS data in the form of a universal grid [6]. Not only does this structure allow one to cover existing raster and vector geoimages of the invariant type, but it also permits the creation of the conditions for differential management of targeted monitoring of the state of natural environment, and for forecasting the of spatio-temporal dynamics of territorial systems using the tools of mathematical modelling in GIS, etc.

This paper elaborates the substantive concept of spatial structure and organization of the territory, based on the hypothesis of existence of a multilevel system of reference points connected by relations of fractal type. Different topologies of point links are induced on this set; each of these topologies corresponds to a particular topic under investigation. Systems of cell structures 
(grid-mosaic) are developed. Each cell is filled with GIS information according to the selected topological model of space organization.

Of some interest are the multilevel multidirectional discrete networks of lineaments (regmatic network) and other geostructures in polysystem models of geographical space [1]. The grid structure of the Earths space organization may be regarded as a natural geoinformation system of the Earth for the construction of the GIS using the invariant space model or a universal grid as a net structure [3], on the basis of the system of reference points. The model of a terrain in the form of a grid structure can be used as a geographical invariant inventory-information basis for the creation of a universal GIS.

\subsection{Natural geoinformation system.}

Let us assume that each point of terrestrial space occupies quite a definite place in the global spatial system, i.e., there is a hierarchical system of spatio-temporal cause-and-effect relationships determining the events at each point at a fixed time. Terrestrial space is highly nonhomogeneous from the geographical point of view. Situations and circumstances are changing from place to place. There are complicated geological manifestations such as tectonic plates, faults, ring formations, geomorphological properties of the relief, landscape diversity of geosystems etc. These heterogeneities are especially noticeable in the analysis of remotely sensed Earth data (RSED). Similar questions were studied in Russia by N.S. Shatsky, V.P. Philosov, O.V. Petrov et al. [1]. A hypothesis of the Earths icosahedral-dodecahedral global system was put forward $[4,5]$, according to which the vertices of two dual Platonic bodies (the icosahedron and the dodecahedron) into which the globe fits correspond to zones of biogeographical activity, and the edges correspond to the lines of tectonic faults. This hypothesis is interesting because points and lines of the geometrical structure have a concrete geographical meaning, i.e., they contain information on the situation.

The primary structure of the Earth is given by the icosahedron (Fig. $1 \mathrm{a}, \mathrm{b}$ ) the faces of which look like an equilateral triangle (Fig. $1 \mathrm{a}, \mathrm{b}$ ). The model consists of 20 triangles completely covering the Earths surface with the triangulation network. An important point is that the scheme of triangulation corresponds to the rectangular graticule of natural parallels and meridians [1]. They pass through the vertices of triangles (nodes of the global network); odd meridians cross at the centre of the base of the triangles. A subsequent fragmentation is carried out by dividing the sides of each rectangular mesh into 6 equal parts (Fig. 2). As a result, the triangle is divided into $6^{2}=36$ small triangles. The vertices of each triangle are crossed by lines of a new rectangular mesh according to the same scheme as in the initial coordination (Fig. $1 \mathrm{c}$ ). Infinitely small fragmentation of rectangular and triangular coordination network is possible due to the same reason.

Hence, a multilevel coordinate system of reference points with fractal properties of self-similarity is specified on the Earths surface where structures of a different level and structures of a different location have a similar organization. In other words, they can be compared with affine transformations (extension, dislocation, and turn). This allows one to compare triangles and rectangles of a different size. Networks of different spatial structure (topology) consisting of elementary cells (triangles, rectangles, hexagons etc.) are specified on a set of base points of the same level. They manifest themselves in different geometrical forms on a terrain [1]. 


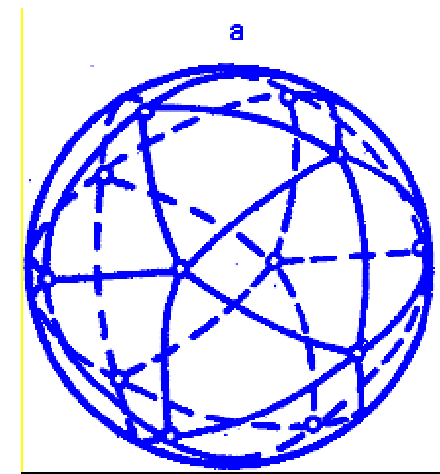

b

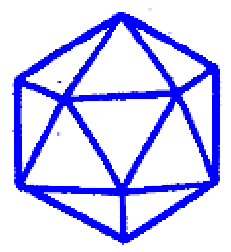

c

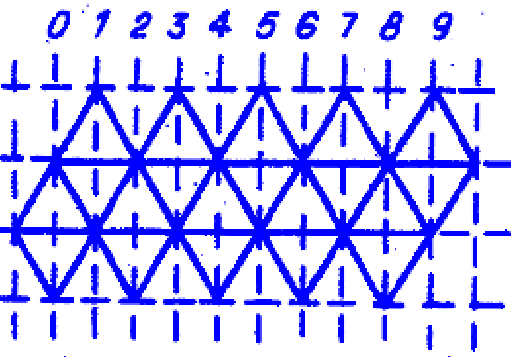

Figure 1: Projections of triangulated earth surface [1]: a - icosahedron projection on the globe surface; $b$ - icosahedron; $c$ - icosahedron in expanded form on the plane; $0-9$ - numbers of triangle pairs and meridians corresponding to them.

It appears that all the points of geospace are differentiated with respect to the discreteness level and the position of nodes of the coordinate grid, while the lines are determined by the coordinates of the nodes which are connected by them. The schematic in Fig. $1 \mathrm{c}$ must be considered an the initial (zero) level. It is followed by the first level of segmentation (Fig. 2), the second, the third, etc. The size of a rectangular horizontal cell of the second level (cf. Fig. $1 \mathrm{c}$ and Fig. 2) is $1 / 360$ of the length of the equator $360=10 * 6 * 6$, i.e. it corresponds to 1 degree or $111 \mathrm{~km}$ $(2 * \pi * 6378 / 360)$. A rough estimate of the cell size along the vertical is $1 /(2 * 3 * 6 * 6)$ of the great circle of the globe, or $185 \mathrm{~km}(2 * \pi * 6356 / 216)$. The triangle side is $111 * 2=222 \mathrm{~km}$, i.e., its height (the corrected size of the triangular vertical cell) is $111 * \sqrt{3}=192 \mathrm{~km}$.

In this manner, a natural grid structure is produced. Each of its reference points has its own individualized geographical code depending both on location property and on the hierarchical level of a given point. In this sense, a grid structure becomes a spatial system of knowledge of geographical content (a natural geoinformation system) which allows one to individualize objects in assessment and predictive computations. The GIS data are all connected with the nodes of a regular grid (reference points), which is convenient for integration of the Earths surface images and information on various spatial processes, 3-D analysis, modelling with the use of cellular automata, and for the construction of other spatial models.

Each cell of the grid structure has the role of: 1) a local system of coordinates; 2) a homogeneous regular elementary unit; 3 ) an elementary information medium; 4) a basic variable structure of database maintenance; 5) a discrete basis for monitoring; 6) an element of the network structure of modelling of the spatio-temporal dynamics; 7) a system of coordination of local phenomena within the cell; 8) a basis for differential local analysis, and 9) a cell of combination of various spatial objects in comparative studies [6].

Grid structures of this kind become a model of invariant structure of the territory reflecting variants of natural topology of the physical-geographical space. A formal differentiation and substantial individualization of space is carried out.

Identifying the Earths multilevel grid structure is an independent and rather complicated task. 
However, its solution is essential for decoding the organization patterns of the Earths space. First and foremost it is necessary to determine the icosahedron orientation, as the vertices of this basic coordination do not necessarily coincide with the Earths geographical poles. Secondly, it is necessary to select an initial point of spatial referenc for estimating the spatial coordinates (parallels and meridians). Thirdly, a model of spatial organization of geographical space must be improved with due regard for the known planetary regularities by introducing discrete (rhythmic) time into the spatial system. Finally, models for calculating the particular parameters, based on multilevel cell spatio-temporal grid structure, i.e. models of spatio-temporal series, will be required.

\subsection{Construction of natural grid-structure model}

The reconstruction of the Earths grid structure has a mutually complementary theoretical and empirical background. The theoretical principles were considered inr [7]. They imply that the initial segmentation of the Earth is specified by the icosahedron, a regular figure with 20 triangular faces; two vertices of the icosahedron coincide with the geographical poles of the Earth $[4,5]$. Such a coordinate hierarchical system will be referred to as the global coordination grid structure (GCGS). A schematic developed view of the Earths surface in the form of such a structure is presented in Figs. 1 and 2.

However, there arises the problem of referencing this network to real geoobjects or identifying geoobjects revealing this structure.

\subsubsection{Theoretical principles.}

Geometrical grid structures of the Earths space are the subject of studies of special through system theory which is based on different branches of science ranging from crystallography to models of the Universe [1]. Polyhedrons consisting of elementary forms (nodes, lines, and triangles) are operational structures of this theory. All polyhedrons can be triangulated they break up into simplexes-elements of a differing dimension (node, line, triangle, tetrahedron 3-simplex). The most common are affine transformations of figures through which polyhedrons are compared or transformed into each other. A.K. Cherkashin showed [8] that an 8-simplex is a limiting one in the 9-D space including particular cases of simplexes of smaller dimension: $C_{9}^{1}=9$ vertices, $C_{9}^{2}=36$ edges, $C_{9}^{3}=84$ flat triangular faces etc.

Investigations of structures are based on the following axioms [1]: 1) the whole diversity of geometrical structures is determined by elements and characteristics of the 8-simplex; 2) all transformations of the 8-simplex are identical, and 3) changes of any polyhedron are affine transformations of its triangulation.

The initial segmentation of the Earth is specified by the icosahedron, a regular figure with 20 triangular faces; two vertices of the icosahedron coincide with the geographical poles of the Earth $[4,5]$. A side of each triangle (see Fig. 1 and 2.1.) is divided into 6 equal parts (successively $2 / 3$ ).

In addition to the triangulation system, a rectangular coordination system is introduced (see Fig. 2) based on the same hierarchy of nodes. Triangulation lines form a local system of coordinates, whereas the lines of coordination produce an orthogonal system. Horizontal (latitudinal) 


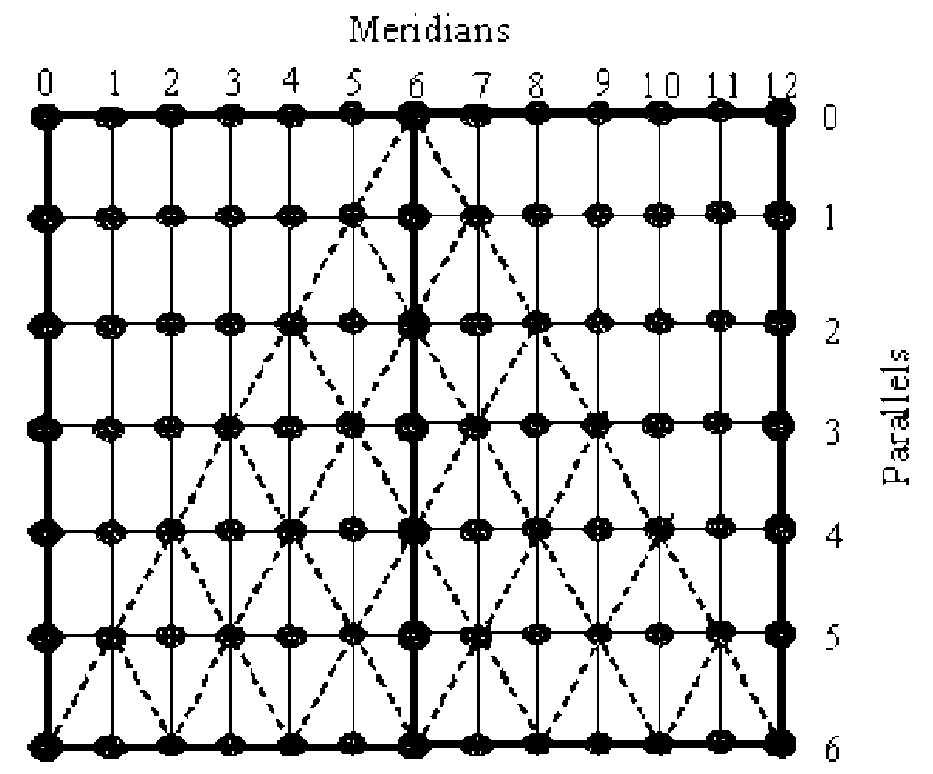

Figure 2: Ratio of triangular and rectangular coordinate grids [1].

and vertical (longitudinal) lines are singled out in the orthogonal system. An expanded coordinate system is formed (see Fig. 1). It involves systems of nodes-analogues : 1) a parallel system forming pentagonal parallels of different lengths from horizontal lines of the ordination system around the poles; 2) a diagonal system of nodes is formed along the lines of the diagonal and orthogonal coordination systems parallel to the sides of the large triangles, and 3) the central system of nodes is formed from the sides and heights of the triangles and crosses the poles of the coordinate system. The GCGS obtained in this manner is tied to the surface of the Earths sphere by 12 global reference points of the vertices of the icosahedron and projected completely onto the sphere describing the icosahedron. When projected, the characteristics of the triangles are distorted: the size of the angles, and the relationships of the sides. Transformations of the figures obey the laws of spherical geometry [7, 8]. For example, 20 large icosahedron triangles-faces are transformed into equal triangles with the side size of $63.4^{\circ}\left(\cos 63.4^{\circ}=\left(\cos 72^{\circ}+\cos 272^{\circ}\right) / \sin 272^{\circ}\right)$ with equal angles of $72^{\circ}=360^{\circ} / 5$ (total $216^{\circ}$ ). Small apical triangles with the side $63.4^{\circ} / 6=10.6^{\circ}$ have an angle of $72^{\circ}$ and two angles of $54.5^{\circ}$ each totalling $181^{\circ}$, which means the transition of the figures from spherical to planar geometry. Moreover, the resulting triangles are not equilateral as is the case with the initial GCGS. This brings up the problems of specifying the rules for GCGS mapping onto the surface a sphere.

It is obvious that when transforming the icosahedron to the spheroicosahedron, there must remain the equality between the sides and angles of the icosahedron triangular faces of the icosahedron $\left(63.4^{\circ}\right.$ and $72^{\circ}$, respectively). In addition,, the rule of dividing the sides into equal parts (into 6) also holds as before. The resulting small triangles which are located in differenet parts of the large triangles are metrically nonequivalent. Let us consider them comparable if their preim- 
ages are comparable in the GCGS, an idealized isomorphic model of the coordinate system on the surface of the Earths sphere (SGCGS). In this connection, the SGCGS characteristics are specified by analogy with the axioms of geometrical structures: 1) the whole diversity of the figures (polyhedrons) is formed on the surface of the Earths sphere and determined by their triangulation as well as by the characteristics of the sphere and the spheroicosahedron; 2) all transformations of the spheroicosahedron are identical, and 3) a change of any figure implies spherical transformations of its triangulation $[7,8]$.

To ensure the connection of the SGCGS with the geographical regularities, it is necessary to tie at least two vertices of the icosahedron to the Earths surface. The geographical pole can be taken as one of these vertices (in the first approximation), whereas the other vertex must be located between the poles. Its form and location must be fixed by the laws formulated in terms of the equations of spherical geometry.

It is known that a spherical triangle is specifiedet by a combination of any one of the three elements [8]: the sides (dihedral angles) a,b,c, and the angles A,B,C (Fig. 3). Therefore, to calculate one element it is necessary to have any other three other elements. Of the 15 possible calculating equations, only 3 equations are independent, e.g., in sine law:

$$
\frac{\sin A}{\sin a}=\frac{\sin B}{\sin b}=\frac{\sin C}{\sin c}=D
$$

Here, a comparison of two opposites is carried out: the sides and the opposite angles of a spherical triangle. And the comparison results in each case turn out to be equivalent and equal to $D$, the individual value for each triangle.

In addition to the comparison equations (2.1), we now specify for any triangle a special value D which determines the basic trianguation of the surface of the sphere: $D=0.91856$ - spherotetrahedron, $D=1$ - spherooctahedron, and $D=1.063314$ - spheroicosahedron. There occurs a proportion of the sides and angles of the basic spehrical triangles, e.g. $\sin 72=1.063314 * \sin 63.4$. The height of the triangles (see Fig. 3) is inferred from the equation $\sin h=\sin 63.4 * \sin 36^{\circ}$ and is $\mathrm{h}=58.28$. The value $31.72=90-h$ isdetermines the location latitude the center of the the base of the large triangle. The system of geographical coordinates to a first approximation is chosen in such a way that the latitude and langitude coincide numerically (they are equal to $31.72^{\circ}$ ) [8].

We suggest that the area of the lower reaches of the Nile in Egypt be taken as such a selected reference point at a global level $[4,5]$. This position corresponds to the centre of the triangle base of the European continental sector continuing symmetrically to the south in the African continental sector, crossing the meridian of $67.72^{\circ}=31.72^{\circ}+36^{\circ}$ in the east proceeding to the Asian sector, with the longitude at the center of the base of $103.72^{\circ}=31.72^{\circ}+72^{\circ}$.

The idea of searching the basic reference point, as suggested by A.K. Cherkashin [8], must be based on the postulate of some harmonic relation between the latitude $\varphi=90^{\circ}-a$ and the longitude A, e.g., from the numerical equality: $\varphi=A$ or $A+a=90^{\circ}$, the expression of complementarity of $a$ and $A$. However, this equality must be formulated similar to (2.1) in terms of the sine of the angles, e.g., as the opposites inverse proportionality relation:

$$
\sin A=K / \sin a \text {. }
$$




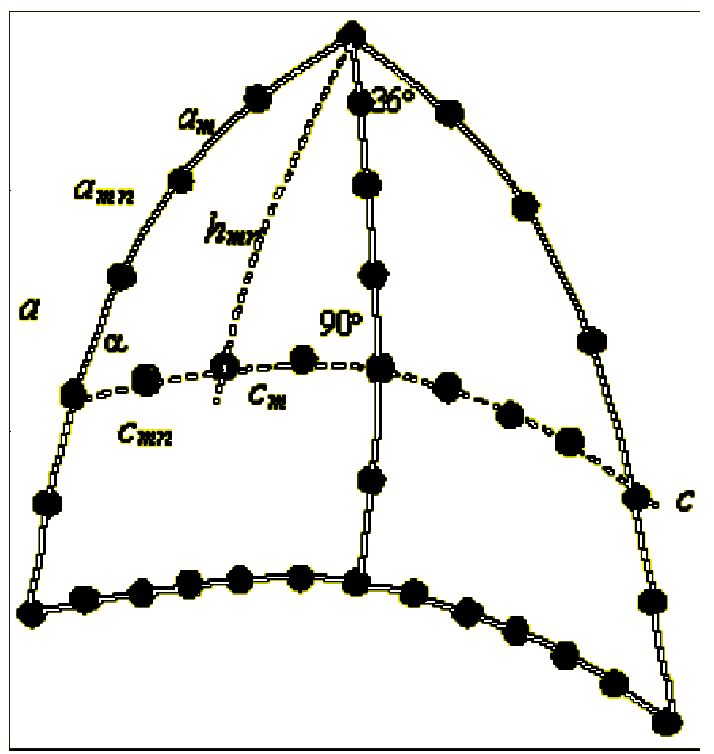

Figure 3: Coordinate calculation of reference points of spherical triangle (explanation in the text) [8].

From this and from (2.2) and (2.1) it follos that $\sin ^{2} A=K D, \sin ^{2} a=K / D \leq 1$ and $K^{2} \leq 1$ - point $\mathrm{D}$ that is determined by the properties of the triangle $\mathrm{D}$. For spherooctahedron we have $D=K=1$, i.e., $\sin A= \pm 1, \sin \alpha= \pm 1$ or $A= \pm \frac{\pi}{2}+2 \pi n, a= \pm \frac{\pi}{2}+2 \pi m$. This relation specifies the Cartesian coordinate system with the origin at the center of the sphere analogous to the system of coordinates which modulates the structure of convective flows at the top level of the Earths asthenosphere [9]. It is represented by three perperdicular large circles, one of which lies on the equator plane and the other two are orthogonal to it and to each other (see Fig. 3). It can be assumed that this octahedral system of coordination is also common to the icosahedron scheme; in particular, in one case, the circumferences (meridians) crossing the poles lie in the Cartesian plane of the octahedral coordinate system. This is approximately the 105-th (Baikal) meridian [1]. In the icosahedral system it crosses the height of the Asian continental triangle and is close to $103.72^{\circ}$. The angle $\sin a=\sin 103.72 / D=0.913622(D=1.063314), a=66.01^{\circ}$ corresponds to this latitude. Hence, $K=0.8875$, which corresponds to angle $62.57^{\circ}$ and is close in its value to the side of the triangular edge of the spheroicosahedron. This suggests that in the relation (2.2) the value $K$ equals the sine of spherical triangle $a_{0}$ of spatially forming figures. This is confirmed for octahedron. Then for the icosahedral structure (2.2) can be recast according to the from (2.1) to

$$
\frac{\sin A}{\sin a_{0}}=\frac{\sin \frac{\pi}{2}}{\sin a}=I_{0} .
$$

When $a_{0}=63.4349^{\circ}$ we obtain $A_{1}= \pm 77.22^{\circ}$ or $A_{2}= \pm 102.78^{\circ}, a= \pm 66.51^{\circ} .90-a$ defines the latitude of the northern and southern tropics. As $\left|A_{1}\right|+\left|A_{2}\right|=180, A_{1}$ and $A_{2}$ specify a certain rule of dividing the hemisphere in order to single out a natural system of geographical coordinates. As the "Baikal meridian" of $103.72^{\circ} \mathrm{EL}$ is very close to $A_{2}$, the existing system of 
geographical coordinates (from Greenwich) is close to the natural one. The problem of determining the longitude of the reference meridian remains open [8].

The global coordinate system is determined by reference points and by first-order lines. The identified positions provide a basis for calculating the different-scale geographical coordinates by the equation

$$
\cos h_{m n}=\cos a_{m n} \cos c_{m n}+\sin a_{m n} \sin c_{m n} \cos \alpha,
$$

where $90-h_{m} n$ is the latitude of the position of the reference point (see Fig. 3) of the n-th triangle; $a_{m n}=a \frac{n}{m}=63,4 \frac{n}{m}, c_{m n}=c \frac{n_{c}}{m_{c}}$, with its vertex to the pole; $m$ is a number segments $(a m)$ of the division of the side of the large triangle (multiple of 6); $a_{m} n$ is the size of the adjacent triangle, and $m_{c}, c_{m}, c_{m} n$ are the same for the base of the adjacent triangles.

According to calculations, the centre of the Asian sector has the geographical coordinates $53.65^{\circ} \mathrm{NL}$ and $103.72^{\circ} \mathrm{EL}$ which is close to the location of Irkutsk. The longitude corresponds to the Baikal meridian [1] of the octahedral and incosahedral system of coordinates.

As is evident from Fig.4, the parallelism of the latitute coordinate lines of the reference points persists in the system of second-order reference points, i.e., the the value of the latitude latitude differs by a constant value. An eastward declination of the direction of the small triangle is observed on the right side of the sector, up to 14 degrees, whereas on the left sidewe see a westward declination. Hence it is possible to indicate the position of central meridians (heights) of the sectors.

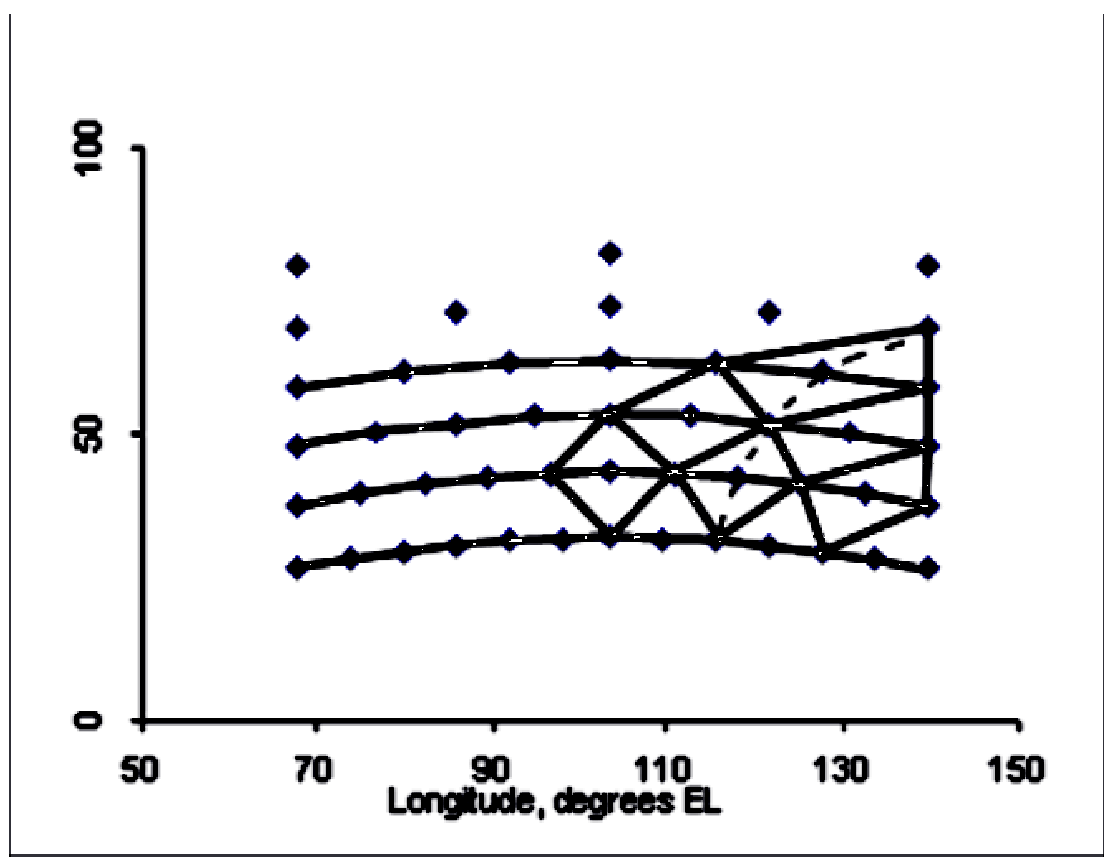

Figure 4: Dependence of latitude on longitude of location of the second order reference points and inclination of triangle orientation [8] . 
These regularities persist in third- and fourth-order systems. The size of a triangular cell and its declination vary with position, but it is relatively constant. In the centre of the Asian sector, for example, the side of the triangle is about $0.32^{\circ}$ or $0.32^{\circ} * 2 \pi R=35.5 \mathrm{~km}(R=6356.8 \mathrm{~km}$ is the polar radius). The cell size of orthogonal grid is $16.4 \times 31.5 \mathrm{~km}^{2}$. The general equation of segmentation of the sides of the triangle with the multiplicity $N_{n}=6$ has the form $L_{\Delta}=$ $\frac{2 \pi R}{360} \frac{N_{n}}{6^{k}} \cdot 63.4$, where $k+1$ is the order of the coordinate system. The sides of the different-order spherical triangles becomes: 1) $7037.9 \mathrm{~km}$, 2) $1173.0 \mathrm{~km}$, 3) $195.5 \mathrm{~km}$, 4) $32.6 \mathrm{~km}, 5) 5.4 \mathrm{~km}, 6$ ) $0.9 \mathrm{~km}$, etc. The triangle corresponds to the sectors of 1) global; 2) regional; 3) subregional; 4) landscape; 4) intra-landscape and 6) local order [8].

\subsubsection{Empirical cartographic analysis}

Maps of regmatic network (network of the earth crust faults lineaments) were used to determine the elements of grid structures. Lineaments generally identify lines of the triangulation (triangular, diagonal) network of grid-structures from which rectangular (orthogonal) coordination is restored [1]. This method was used in the cartographic analysis of faults in Pribaikalye (Baikal region) to identify systems of reference points and topology of grid structures. In this study we pursued two objectives: 1) to estimate the whole network of faults and 2) to analyze characteristics of faults in granitoid fields as well as in the homogenous geological structure.

The area of Southern East Siberia (the Irkutsk Region and Pribaikalye) as investigated first. The faults and the river system were used as the reference objects (as the indicator of faults and relief structure). The map of faults using Resource-F space images in relative coordinates was compiled by the Institute of the Earths Crust of the Siberian Branch of the Russian Academy of Sciences [10]. This map was vectorized for visual analysis in the Corel Draw graphic editor. It contained faults proper and their special geological symbols in one vector layer. Therefore, it had to be edited for the analysis in GIS ArcView. The GIS analysis of the whole of the selected territory showed that the basic distribution of fault directions are 45 degrees, whereas the medium size of triangular cells determining the grid structure (see Fig. 1) makes up $35.3 \mathrm{~km}$. Points of the support grid structure are calculated for different-order networks and were overlapped with the fault network for further expedition surveys of these territories in order to reveal specific characteristics of the landscape.

At the same time, this analysis was insufficient because only linear geological objects were used. Ring structures from the fault map of Pribaikalye were additionally used in subsequent studies. This analysis showed that reference points of the computational grid structure mainly coincide with elliptical fragments of ring structures or with structure centres in the form of circles. The higher-order network are included in the larger-size structures or the system of structures formed by smaller fragments of rings and faults. Figure 66 presents the schematic of the Pribaikalye territory with ring structures marked in the map of faults of the Earths in Southern East Siberia and the reference points of the computational grid-structure.

The peculiar feature of the study territory is that the middle part of Predbaikalye is covered in a thick sedimentary mantle, which makes it difficult to identify faults and distorts the structure of the river network. Therefore, studies of the fault network in the homogenous geological rock are 


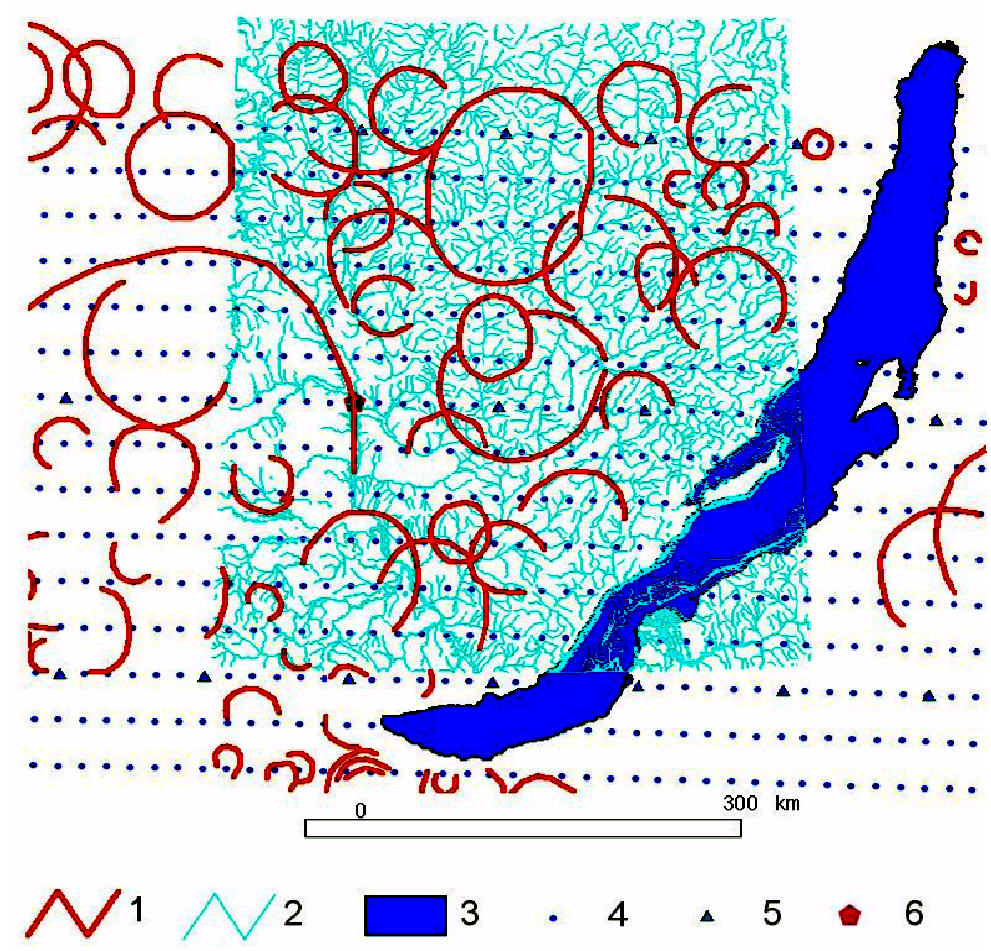

Figure 5: Scheme of ring structures (1), hydronetwork (2 - hydronetwork of topographic map sheet No. 48, 3 - Lake Baikal) and reference points of the grid-structure (4 - 4th, 5 - 3rd, and 6 2nd orders) of Pribaikalye.

of interest for verification of results. In geologists opinion, granites are the most homogenous and amorphous rock without lamination. Thus the fault networks were analyzed in granitoid fields. Faults in the GIS ArcView terms are represented as lines (linear theme), while granitoid fields are represented as polygons (polygonal theme). The technology of this procedure is as follows:

1. Adjustment of the most detailed vector map of Pribaikalye faults [10] and raster map of faults of Southern East Siberia (scale 1:1 500 000) to common geographical coordinates was carried out with GIS instruments.

2. Granitoid fields as polygons were vectorized from the fault map of Southern East Siberia.

3. Faults belonging only to granitoid fields were singled out with the help polygonal theme of granites from the linear theme of faults of the Pribaikalye vector map.

4. Data obtained were edited: additional objects connected with the specific character of presentation of geological structures were removed. 


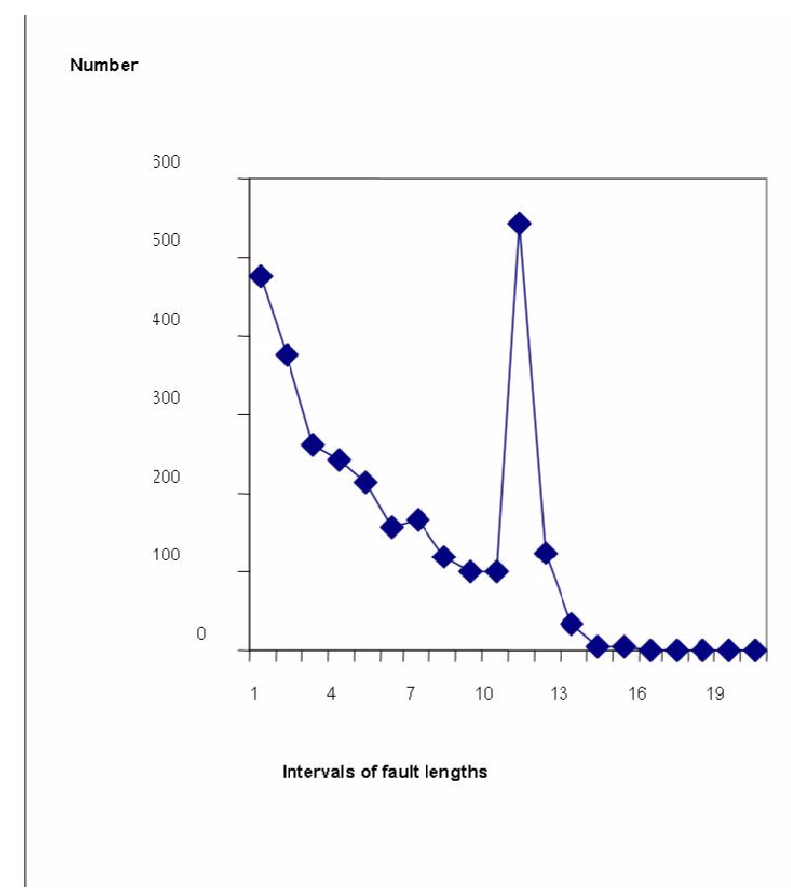

Figure 6: Fault occurrence of different length in granitoid fields.

5. Fault lengths were calculated with special ArcView scripts and presented in tables.

6. Nodes, points of fault bending of some linear segments, were singled out from fault lines. Angles of deviation from the north-south segments of faults were calculated from the ratio of node coordinates.

7. The summary attributive table was compiled with node coordinates, segment lengths and their directions in degrees.

8. The data characterizing the length and directions of the faults were analyzed with MS Excel.

Twenty groups (intervals) of lengths were selected for the analysis of the fault lengths: 10 intervals from 0 to $10 \mathrm{~km}(1 \mathrm{~km}$ each), and 10 intervals ranging from $\mathrm{km} 10$ to 100 at steps of $10 \mathrm{~km}$. According to the GIS analysis of the data of the map (Fig. 7), the greatest number of faults was within the first interval, up to $1 \mathrm{~km}$. The frequency of different-size fault occurrence gradually decreases towards the $10^{\text {th }}$ interval (up to $10 \mathrm{~km}$ ). The maximum of occurrence is observed between intervals 10-13 (with the size varying from 10 to $30 \mathrm{~km}$ ). Only 12 lineaments are recorded within the intervals $14-40$, i.e. $10-30 \mathrm{~km}$.

Thus the peculiar characteristics of fault lengths are observed at $\mathrm{km} 1, \mathrm{~km} \mathrm{4-7,} \mathrm{and} \mathrm{km} 30$. This corresponds to computational grid structures (the sides of triangular cells) of local, intra-landscape and landscape order (see 2.2.1). It is impossible to determine the parameters of larger cells in this experiment because of the limited size of granitoid fields of this territory. 


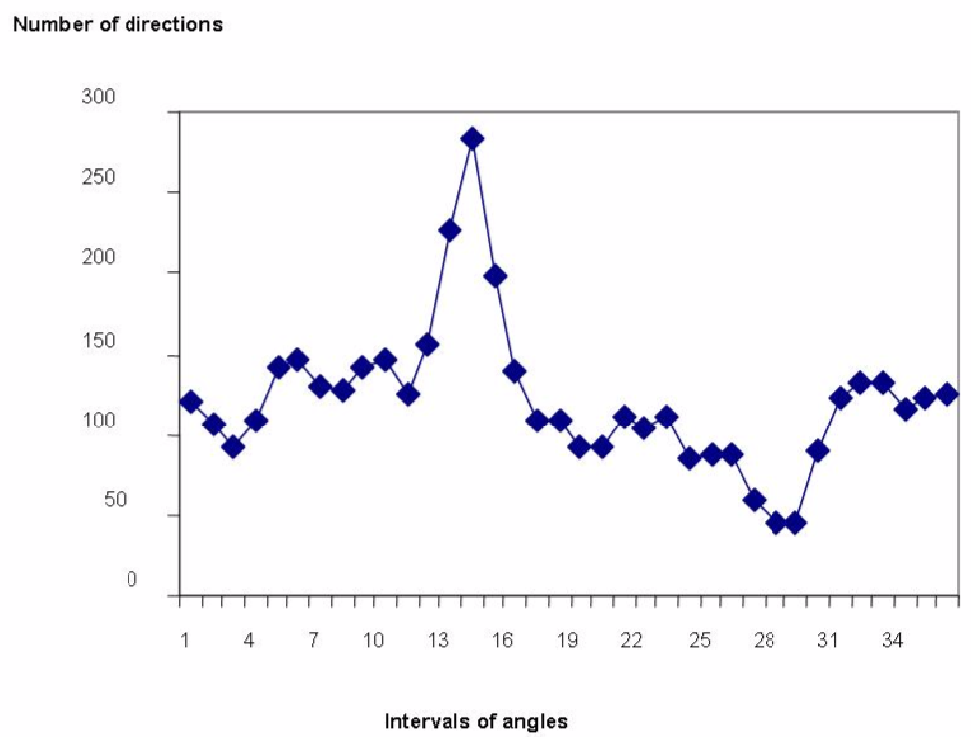

Figure 7: Occurrence of faults of different directions in granitoid fields.

It was possible to distinguish 36 intervals of 10 degrees each for GIS analysis of fault direction $(360 / 36=10)$ with the deviation ranging from -180 to +180 degrees from the 105 -th meridian. Most of the faults are inclined towards the meridian at an angle of 45 degrees (Fig. 7) (Fig. 7). A sudden increase was observed in the deviations to the east and west from the meridian: local maxima in intervals 4-5 (about 45 degrees), in 21-23 $(225-180=45)$, in 31-32 $(360-315=45)$ and global maximum in intervals 13-15 (about 135 degrees $180-135=45$ ).

Based on these parameters, the diagonal network of faults setting a triangulation structure of the territory was singled out in the map of geological faults (Fig. 8). Basic lines go along the Angarsk fault and the north-western coast of Lake Baikal marking out the Baikal rift zone. The coordination network has rhomboid cells of the 4-th level (line 7, Fig.8). The average size of the sides $(35.3 \mathrm{~km})$ is in agreement with the computational values $(32.6 \mathrm{~km})$. The coordinate network has a slight eastern inclination of up to 5. A comparative analysis of the networks was carried out for the case of the overlapping (in GIS) of the fault network, hydrological network and the network of reference points. It was concluded that their best overlapping occurs if the reference meridian $103.72^{\circ} \mathrm{E}$ is shifted to $103.38^{\circ} \mathrm{E}$. There are no empirical reasons for the shifting of the network along the latitude. The orientations and, partly, the size of lineaments correspond to the directions and size of the lines connecting the reference points. 


\section{Conclusion}

The analysis showed that there are triangular, rhomboid (from contiguous triangles) and orthogonal structures connected with the triangulation network having the cell side size (triangle base) of 35.5 $\mathrm{km}$ and the orthogonal cell size of $16.4 * 31.5 \mathrm{~km}$. These cells represent a characteristic set of 4th-order cells. Moreover, nested structures of higher fraction order (on average $17.5 \mathrm{~km}$ ) and up to $1 \mathrm{~km}$ are observed, as well as structures of regional and global levels.

The preferred orientation of faults as regards the 105-th meridian (about 45 degrees) is preserved in the total sample of lineaments around Pribaikalye and in granitoid fields.

The studies conducted by considering an example of Pribaikalye have validated the hypothesis of icosahedron grid structures. It can serve as the framework for the construction of natural GIS based on the triangulation network as calculated by A.K. Cherkashin and coordinated with the corresponding rectangular network of a different hierarchy with fractal characteristics.

\section{References}

[1] A. Cherkashin. Polysystem analysis and synthesis. Application in geography. Nauka, Novosibirsk, 1997, 502 p.

[2] A. Cherkashin, A. Kitov, I. Bychkov et al. Geoinformation system of territory management . Izd-vo Institute of Geography SB RAS, Irkutsk, 2002, 151 p.

[3] A. Kitov. Computer analysis and synthesis of geoimages. Izd-vo SB RAS, Novosibirsk, 2000, $220 \mathrm{p}$.

[4] N. Goncharov, V. Makarov, V. Morozov. Power structure of the Earth and organization of nature-conservative measures. Nature-conservative measures in landscapes (development and organization), (1982), Izd-vo USSR Geographical Society, Moscow, 113-124.

[5] N. Goncharov, V. Makarov, V. Morozov. Is the Earth a big crystal? Zakharov, Moscow, 2005, $224 \mathrm{p}$.

[6] A. Kitov, A. Cherkashin. Grid-structures as an invariant basis of geoinformation system realization. InterCarto/InterGIS 10: Sustainable Development of Territories: Geoinformation Support and Practical Experience. International Cartographic Association, VladivostokChanchun, 2004, 17-24.

[7] A. Kitov, A. Cherkashin. Recovery of regular grid-structures for GIS of Pribaikalye from lineament map. InterCarto-InterGIS 12: Sustainable Development of Territories: GIS Territory and Practical Experience. Vol. 1. International Cartographic Association, Kaliningrad-Berlin, 2006, 37-45.

[8] A. Kitov, A. Cherkashin. Empirical cartographic analysis. Geographical studies of Siberia. Vol. 4. Polysystem Theme Cartography. Academicheskoye Izd-vo "Geo", Novosibirsk, 2007, 275-279. 
[9] T. Irvine. Global convection framework: concepts of symmetry, stratification and system of the Earth's dynamic structure Econ. Geol., 84 (1989), No. 8, 2059-2114.

[10] K.G. Levi, A.V. Arzhannikova, V.Yu. Buddo, P.G. Kirillov , A.V. Lukhnev, A.I. Miroshnichenko, V.V. Ruzhich, V.A. Sankov Current dynamics of the Baikal rift Exploration and Conservation of Mineral Resources, (1997), No. 1, 10-20. 


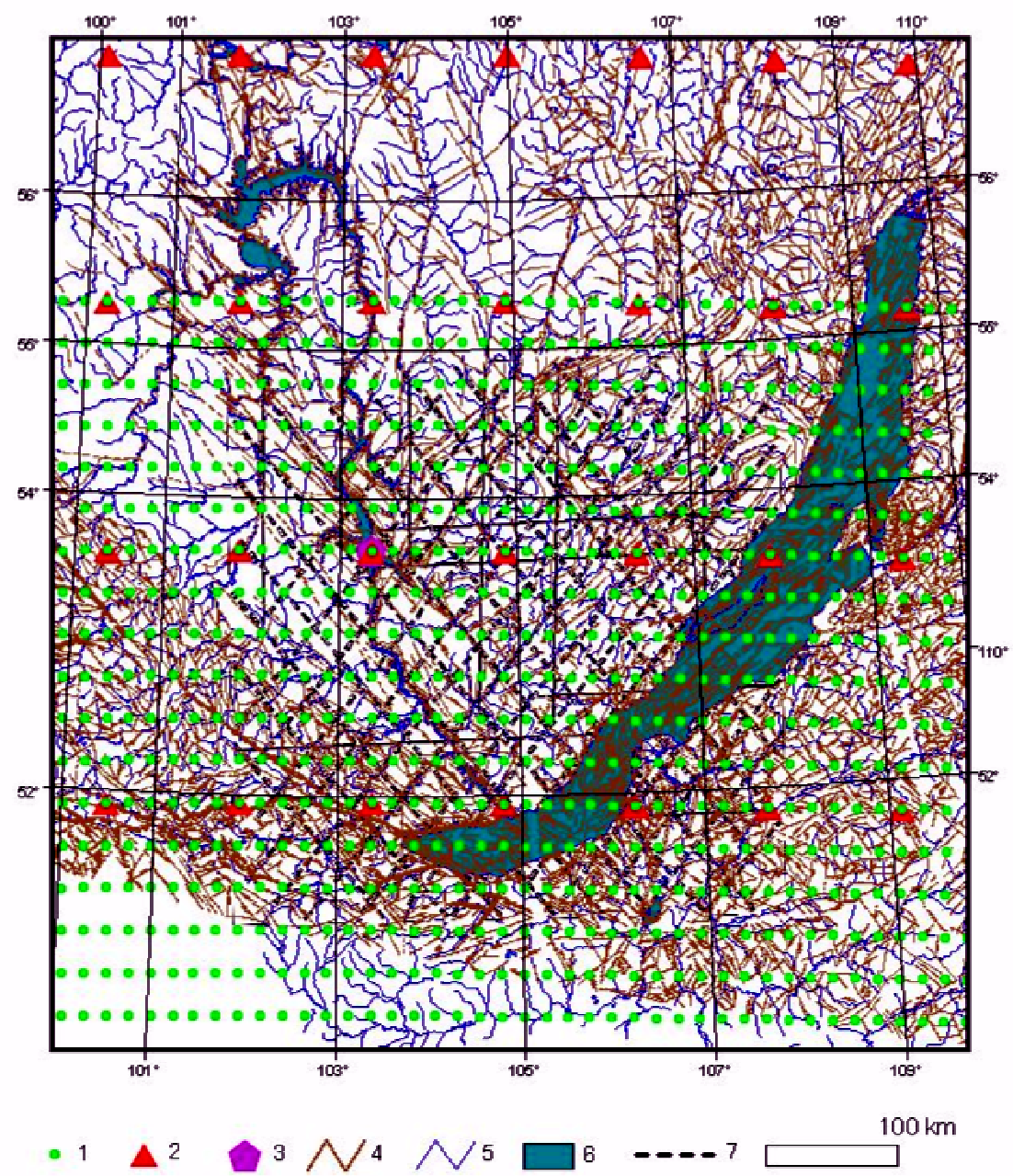

Figure 8: Cartographic comparison in position of river, regmatic and coordinate networks. Gridstructures: 1 - topological (4th order); 2 - regional (3th order); 3 - calculation centre of the Asian continental sector; 4 - network of lineaments; 5 - hydronetwork; 6 - lakes and reservoirs; 7 diagonal systems of faults. 